\title{
UN CASO DE APLICACIÓN DE LA DERMATOLOGÍA INTEGRAL
}

\author{
Luis Antonio Castro ${ }^{1}$, Angela María Bedora ${ }^{2}$ \\ ${ }^{1}$ Especialista Dermatología, Inmunodermatología. Hospital Militar Central, \\ Universidad Militar Nueva Granada \\ ${ }^{2}$ Residente de Dermatología. Hospital Militar Central, Universidad Militar Nueva Granada \\ CORRESPONDENCIA: ANGELA_BEDOYA2000@YAHOO.COM \\ Recibido: JULIO 2 DE 2013 ACEPTADo: OCTUBRE 11 DE 2013
}

\begin{abstract}
Resumen
La sarcoidosis es una enfermedad granulomatosa multisistémica de etiología desconocida. Presentamos el caso de una mujer que consulta por la preocupación estética de una lesión en dorso nasal. La exploración clínica lleva a sospechar de una enfermedad granulomatosa, por lo cual se lleva a biopsia y confirmación de su patología. La sarcoidosis es un trastorno crónico, que afecta múltiples órganos, su incidencia es variable, pero es mayor en países industrializados y se presenta en su mayoría en mujeres entre 20 y 40 años de edad. De las manifestaciones sistémicas, la más frecuente es la pulmonar, mientras que la afectación cutánea oscila entre 20-35\%. El diagnóstico se establece con los hallazgos clínico-radiológicos apoyados por la evidencia histopatológica de granulomas no caseificantes, aunque estos últimos no son específicos, por lo que deben excluirse otros procesos.
\end{abstract}

Palabras clave: Sarcoidosis, cutáneo, granulomas.

\section{A CASE OF APPLICATION OF INTEGRAL DERMATOLOGY}

\begin{abstract}
Sarcoidosis is a multifactorial granulomatous disease of unknown etiology. We present a case of a woman who presents with cosmetic concerns of a lesion on the dorsum of the nose. The clinical exploration raises suspicion of granulomatous disease, for which reason a biopsy is done confirming her pathology. Sarcoidosis is a chronic disorder that affects multiple organs. Its incidence is unknown but is more frequent in industrialized countries, and majorly affects women between 20-40 years of age. Among its multiple manifestations, pulmonary symptoms are the most frequent, meanwhile the cutaneous manifestations are only present in $20-35 \%$ of the cases. The diagnosis is made by clinical-radiographic findings associated to histopathological findings of noncaseating granulomas, even though these are not specific to the disease and other processes must be ruled out.
\end{abstract}

Key words: Sarcoidosis, cutaneous, granulomas. 


\title{
UM CASO DE APLICAÇÃO DA DERMATOLOGIA INTEGRAL
}

\begin{abstract}
Resumo
A sarcoidose é uma doença granulomatosa multisistêmica de etiologia desconhecida. Apresentamos o caso de uma mulher que consulta pela preocupação estética de uma lesão em dorso nasal. A exploração clínica leva a suspeitar de uma doença granulomatosa, pelo qual se leva a biopsia e confirmação de sua patologia. A sarcoidoseé um transtorno crônico, que afeta múltiplos órgãos. A sua incidênciaé variável, mas é maior em países industrializados e tempredileção porque apresenta em sua maioria nas mulheres entre 20 e 40 anos de idade. Das manifestações sistêmicas, a mais frequente é a pulmonar, por enquanto que a afetação cutânea oscila entre $20-35 \%$. O diagnóstico se estabelece com as descobertas clínico-radiológicas apoiadas pela evidência histopatológica de granulomas não caseificantes, porémestes últimos não são específicos, pelo que devem se excluir outros processos.
\end{abstract}

Palavras chave: Sarcoidose, cutâneo, granulomas.

Introducción: La historia moderna de la sarcoidosis, como una enfermedad multisistémica, enigmática, va hasta 1899, cuando el pionero dermatólogo noruego Caesar Boeck acuñó el término para describir nódulos cutáneos caracterizados por focos definidos de "células epitelioides con núcleos grandes pálidos y también pocas células gigantes", pensando que era un sarcoma, y le llamó "sarcoide benigno múltiple de la piel"(1).

La sarcoidosis es una enfermedad sistémica de causa desconocida constituida por granulomas no caseosos, donde no se encuentran microorganismos, ni partículas extrañas (2). Es una enfermedad multisistémica que involucra los pulmones, ganglios, hígado, corazón, ojos y piel (3), y en la cual se estima que el compromiso cutáneo está presente en el $25 \%$ de los casos (4). Sus manifestaciones clínicas son variadas y es considerado uno de los grandes imitadores, pero por simplicidad las lesiones se dividen en específicas (en la cual se observan granulomas) típicas y atípicas y en inespecíficas (en la cual no se observan granulomas). Dentro de las lesiones específicas se encuentran, siendo la más frecuente la erupción maculopapular, microtubérculos, macrotubérculos, placas persistentes, lupus pernio y nódulos subcutáneos indoloros, llamado sarcoidosis de Darier-Roussy (5). En las específicas atípicas, se encuentran la sarcoidosis cicatrizal, formas psoriasiformes, eritrodérmicas, ictiosiformes, eritematosas e hipo-hiperpigmentadas. El eritema nodoso es la manifestación inespecífica más frecuente. Se presenta como nódulos dolorosos en las extremidades hasta en el $48 \%$ de los pacientes y muestra un predominio en mujeres de todo el mundo(6).
En las mujeres la sarcoidosis es más frecuente que en los hombres y en los adultos de menos de 40 años. Un segundo pico de incidencia se reporta en las mujeres mayores de 50 años. Es más prevalente en escandinavos, daneses y afro-norteamericanos, con una prevalencia de hasta 64/100,000 (7,15). Las manifestaciones varían dependiendo de la etnia de la persona, siendo lupus pernio más frecuente en personas de la zona de puerto rico, comparado con afro-americanos quien presentan más frecuentemente eritema nodoso(8).

La etiología aún es desconocida y el evento se desarrolla por varios factores genéticos y ambientales o la combinación de ambos (9), que hacen al individuo susceptible o no de la enfermedad. Esa predisposición y susceptibilidad conllevan a una respuesta inflamatoria exagerada a ciertas exposiciones ambientales, con activación inmune local y formación de granuloma. La característica cardinal es la formación del granuloma y su mantenimiento es el resultado de la presencia de células T CD4+ que interactúan con células presentadoras de antígenos.

Entre los factores que se mencionan está la heredabilidad, debido a que hay más probabilidades de padecerla cuando hay parientes cercanos con la misma enfermedad. Este factor estimado en gemelos es del $66 \%$.

Caso clínico: Mujer de 53 años que consulta por una preocupación estética, ella nota en su rostro la aparición de una única pápula eritematosa de $4 \mathrm{~mm}$ de diámetro, infiltrante en dorso nasal de un mes de evolución (figura 1 a y b). En razón de las características 
clínicas de la lesión se decide tomar biopsia de piel y se le explica a la paciente la necesidad de realizar dicho procedimiento ante la sospecha de una lesión granulomatosa, el reporte histopatológico demostró una dermatitis granulomatosa con granulomas de tipo sarcoidal (figura 2).

Se solicitaron los estudio paraclínicos para descartar compromiso sarcoidal sistémico, como positivo los rayos $\mathrm{X}$ de tórax demostraron adenopatías parahiliares y un nódulo pulmonar. Posteriormente como estudio complementario se le realiza TAC torácico con contraste, que reporta ganglios hiliares bilaterales de 8 y 10 mm, y como hallazgo incidental un nódulo pulmonar en língula de 16x14 mm (figura 3). Se realiza la impresión diagnostica de neoplasia pulmonar, y es llevada a cirugía donde se le realizó una biopsia de masa parahiliar, el resultado de la biopsia señala sarcoidosis y una lobectomía superior izquierda indica hamartoma pulmonar. La paciente presentó una recuperación quirúrgica satisfactoria y le fue iniciado prednisona a $1 \mathrm{mg}$ por kilogramo de peso, con posterior reducción progresiva de los mismos por 6 meses con mejoría clínica e imagenológica de las lesiones.

Discusión: La sarcoidosis es una enfermedad de etiología desconocida. Estudios recientes que emplean análisis genómico, han permitido reconocer el gen BTNL 2 como un marcador de susceptibilidad a sarcoidosis y la identificación de la peroxidasa-catalasa micobacteriana como un potencial "antígeno sarcoidal" que conduce a la formación del granuloma $(6,15,17)$. Estos estudios se efectuaron en sarcoidosis pulmonar. Las enfermedades granulomatosas se consideran el resultado de un estímulo antigénico desconocido; ello conduce a la activación de las células $\mathrm{T}$ que genera la liberación de citoquinas. El antígeno desconocido es fagocitado por las células presentadoras de antígenos (CPA) o células dendríticas. Luego, el material antigénico procesado es transportado a la superficie, donde activa a las células T CD4+, esto lleva a la producción de citoquinas Th 1: INF- $\gamma$, IL-2 e IL-12. Se observa incremento de TNF- $\alpha$ que aumenta, a su vez, la secreción de citoquinas que elevan el reclutamiento de macrófagos en el granuloma $(10,11,12)$.

En un $25 \%$ de casos de sarcoidosis aparecen lesiones cutáneas (11), generalmente lo hacen al inicio de la enfermedad y no es infrecuente realizar el diagnóstico de esta entidad en la consulta dermatológica. Marcoval y colaboradores sugieren que todos aquellos pacientes con granulomas cutáneos de tipo sarcoidal deben ser evaluados para descartar la presencia de una sarcoidosis sistémica. La ausencia de afectación sistémica tampoco excluye el diagnóstico de sarcoidosis; de hecho, existen formas de afectación exclusivamente cutánea en el $27 \%$ de la población blanca, que son indistinguibles de las lesiones cutáneas de las formas sistémicas. Ahora bien, en todos aquellos pacientes con lesiones cutáneas sin afectación sistémica, debe realizarse un tamizaje de sarcoidosis sistémica de forma periódica, pues la sarcoidosis sistémica puede aparecer en cualquier momento de la evolución $(13,18)$.

El diagnóstico se establece, cuando los hallazgos clínicos y radiológicos se apoyan en la evidencia histológica de granulomas no caseificantes, diseminados en uno o más tejidos y exclusión de otras enfermedades. Debe incluir una biopsia, rayos $\mathrm{X}$ de tórax, TAC pulmonar, función pulmonar, cuadro hemático, medición de enzima convertidora de angiotensina.

En más de dos tercios de los pacientes, en el término de diez años, se produce remisión espontánea de la enfermedad con poca o ninguna consecuencia. Pero desafortunadamente la enfermedad persiste hasta en un tercio de los pacientes, dando un deterioro de la función de múltiples órganos. A pesar de los múltiples efectos secundarios a largo plazo, el tratamiento principal siguen siendo los corticosteroides, que suprimen la inflamación a través de la inhibición de la síntesis de citocinas. En general, se requieren de 6 a 9 meses de tratamiento con una dosis de $30-40 \mathrm{mg} /$ día de prednisona cada 2 semanas, disminuyendo $5 \mathrm{mg} /$ día hasta llegar a 10 a $20 \mathrm{mg} /$ día, dosis que se mantendrá por algunos meses. Pero aún no hay un consenso definitivo sobre las dosis y tiempo de uso $(14,15)$.

Los agentes que modulan las acciones clave de las células CD4 TH1, pueden tener efectos beneficiosos para el tratamiento de la sarcoidosis, sin los efectos deletéreos a largo plazo de los corticoesteroides (19).

La pentoxifilina que es un inhibidor selectivo de la fosfodiesterasa, posee propiedades antiinflamatorias conocidas y se ha demostrado que inhibe al TNF. En los Institutos Nacionales de Salud de USA, se realizó un estudio aleatorizado, doble ciego, controlado con placebo, para determinar si la pentoxifilina podría ser un tratamiento alternativo a la prednisona. En este estudio se comprobó menos reactivaciones en los pacientes que recibieron pentoxifilina, comparados 
con aquellos que recibieron placebo. Por otra parte, el grupo pentoxifilina utilizó menos prednisona, lo que sugiere un efecto ahorrador del esteroide (20).

Las estatinas disminuyen los niveles de colesterol y tienen propiedades inmunomoduladoras, regulan los factores de transcripción importantes en la inflamación, incluyendo el NF- $\kappa \beta$ la proteína activadora 1 y los receptores activados por el proliferador de peroxisomas, y disminuyen la expresión de las moléculas coestimuladoras y la activación de las células dendríticas. También suprimen la diferenciación de CD4 TH1, limitan la producción de quimiocinas, por lo cual se sugiere que podrían tener un efecto antiinflamatorio y ser utilizado en el tratamiento de la sarcoidosis (21).

El metotrexato, un antimetabolito, interfiere la síntesis de ADN y la reparación y la replicación celular a través de inhibición reversible de la dihidrofolatoreductasa. También inhibe otra enzima, lo cual se traduce en un aumento de las concentraciones de adenosina (un potente mediador antiinflamatorio). El metotrexato inhibe las respuestas inmunes mediadas por células y ha sido utilizado para tratar otras enfermedades granulomatosas. Se desconoce el mecanismo de acción exacto del metotrexato en la sarcoidosis, pero se cree que su acción puede radicar en la supresión del TNF y el aumento de la adenosina extracelular. El FNT es una citocina importante en la inflamación granulomatosa y ejerce su acción en múltiples sitios celulares y subcelulares (22).

Existen otros medicamentos que han sido utilizados para sarcoidosis cutáneas como los supresores del FNT, que parecen ser críticos en la formación de granulomas. Uno de los cuales seria la Talidomida a dosis de 100 $\mathrm{mg}$ al día, con un efecto adverso muy frecuente, la neuropatía periférica. Otro medicamento más reciente es el Infliximabun, anticuerpo monoclonal quimérico contra el FNT (23).

Conclusión: Traemos una paciente quien consulta por la implicación estética de una lesión mínima en cara, que de acuerdo con su apariencia clínica, generó el diagnóstico de sarcoidosis cutánea sistémica, y el hallazgo incidental de un hamartoma pulmonar que finalizó en lobectomía pulmonar.

El órgano más afectado por la sarcoidosis es el pulmón, sin embargo, también es común la afección a la piel, incluso las primeras manifestaciones de la sarcoidosis descritas en la literatura son las lesiones dermatológicas. Se cree que para el desarrollo de la enfermedad se requiere cierta predisposición o susceptibilidad genética, así como la interacción de tres factores: exposición a antígenos no específicos, inmunidad celular adquirida ante dicho antígeno y aparición de una respuesta inmune, inflamatoria e inespecífica. Debido a que la sintomatología de la sarcoidosis es completamente inespecífica y a que en general, puede afectar diversos órganos, el diagnóstico suele retrasarse meses e incluso años. Entre 30 y $60 \%$ de los pacientes con sarcoidosis pulmonar son asintomáticos, como es el caso de la paciente observada. Consecuentemente, el diagnóstico de sarcoidosis debe sospecharse en pacientes con lesiones en piel y en presencia y/o ausencia de síntomas sistémicos. La observación de este caso y sus conclusiones, refuerzan el enfoque integral y la importancia crucial del dermatólogo y la piel como manifestación de compromiso sistémico de diversas enfermedades. De esta forma podemos observar que el conocimiento y la sospecha sirven para un diagnóstico adecuado, siendo de vital importancia para mejorar la evolución clínica y el pronóstico de nuestros pacientes.

Figura 1. (A) Pápula eritematosa infiltrada de $4 \mathrm{~mm}$ en dorso nasal. (B) Ampliación de la lesión.
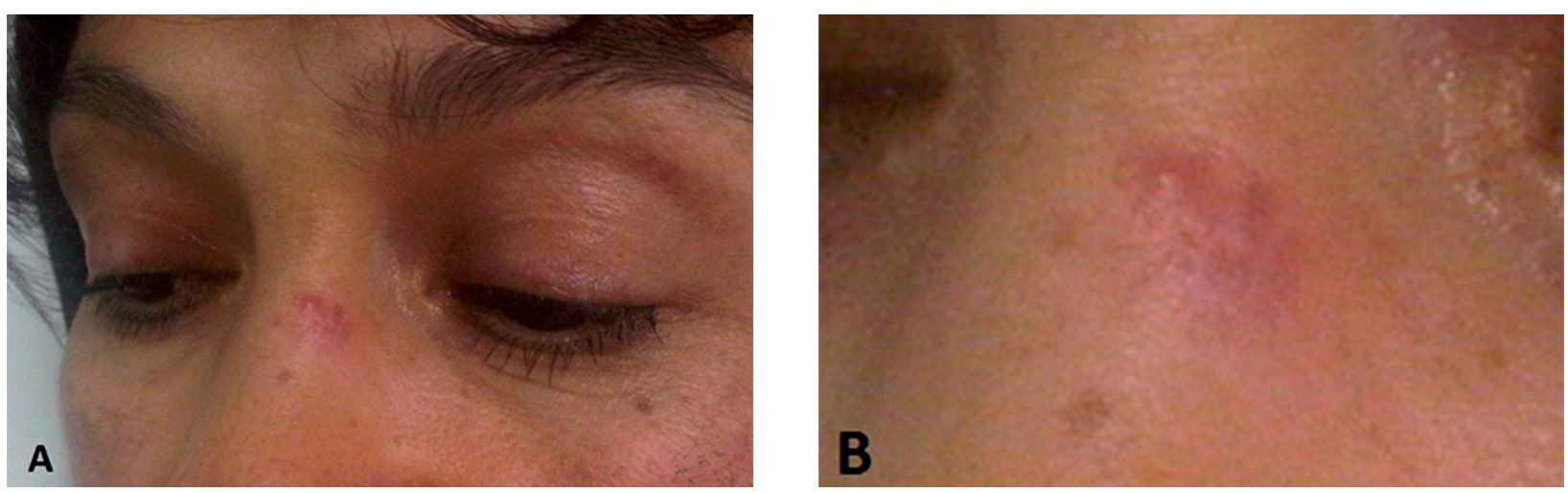
Figura 2. Estudio histopatológico de biopsia de piel, que muestra dermatitis granulomatosa con granulomas tipo sarcoidal (recuadro).

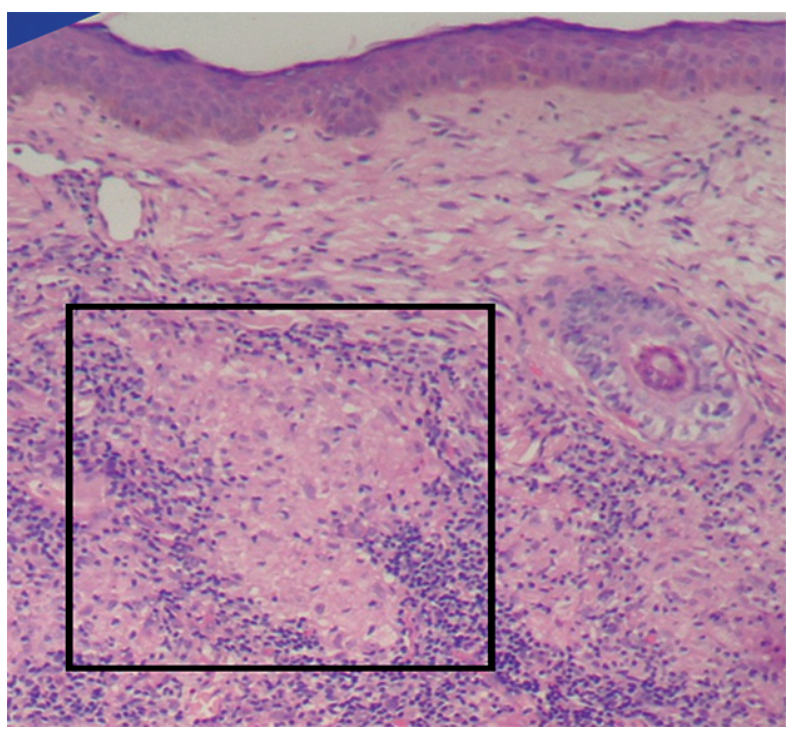

Figura 3. (A) TAC torácico en el que se aprecian nódulos parahiliares bilaterales. (B) RMN torácica con medición del nódulo hamartomatoso en língula. Énfasis en medición (recuadro).
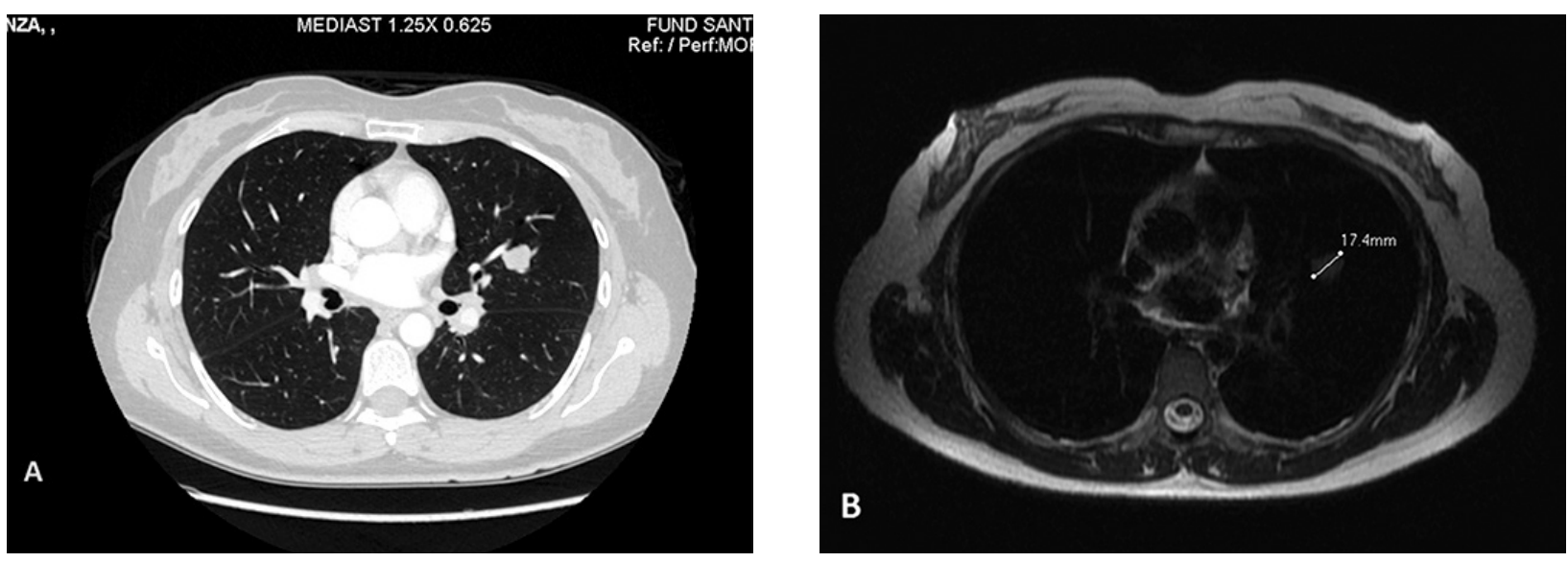

\section{Referencias}

1. Boeck C. Multiple benignsarcoid of the skin. J Cutan Genitourin Did. 1899; 17: 543-550.

2. Lannuzzi M, Rybicki BA, Teirstein AS. Sarcoidosis. New England Journal of Medicine. 2007; 357 (21-22): 2153-65.

3. Raghunatha R, Shashi K, Harish MR. Cutaneous sarcoidosis A great masquerader : A report of three interesting cases. Indian J Dermatol. 2011; 56(5): 568-572.

4. Newman LS, Rose CS, Maier LA. Sarcoidosis. N Engl J Med. 1997; 336: 1224-1234.

5. Rabinowitz OL, Zaim MT. A clinicopathologic approach togranulomatous dermatoses. J Am Acad Dermatol. 1996; 35 : 588-600.

6. De las Heras MH, Jaén OP. Sarcoidosis cutánea. JANO. 2009; 1 (739): 34-36.

7. James WD, Berger TG, Elston DM. Editores. Sarcoidosis. Andrew's diseases of the skin. $10^{\mathrm{a}}$ ed. Canada: Saunders Elsevier; 2006. p. 708-14.

8. Hamzeh N. Sarcoidosis review article. Medical Clinics of North America. 2011; 95(6):1223-1234.

9. Vourlekis JS, Sawyer RT, Newman LS. Sarcoidosis: developmentsin etiology, immunology, and therapeutics. AdvIntern Med. 2000; 45: 209.

10. Locurscio A, Morichelli M, Canonicci L, Fiducia ML, Rodríguez A, Manzur ME. Sarcoidosis cutánea. Arch Argent Dermatol. 2005; 55: 229-237. 
11. Noor A, Knox K. Immunopathogenesis of sarcoidosis. Clinical Dermatol. 2007; 25: 250-258.

12. Gurrieri C, Bortoli M, Brunetta E, Piazza F, Agostini C. Cytokines, chemokines and other biomolecular markers in sarcoidosis. Sarcoidosis Vasculitis and Diffuse. LungDiseases. 2005; 22(1): S9-S14.

13. Song Z, Marzilli L, Greenlee B, Chen E, Silver R, Askin F, et al. Mycobacterial catalase-peroxidase is a tissue antigen and target of the adaptive immune response in systemic sarcoidosis. $\mathrm{J}$ ExpMed. 2005; 201(5): 755-767.

14. Badgwell C, Rosen T. Cutaneous sarcoidosis therapy updated. J Am Acad Dermatol. 2007; 56: 69-83.

15. Sanz T, Aragües M, Fernández J, Fraga J, García A. Sarcoidosis subcutánea. Actas. Dermosifiliogrina. 2000; 91: 321-6.

16. Veien NK, Stahl D, Brodthagen H. Cutaneous sarcoidosis in caucasians. J Am Acad Dermatol. 1987; 16: 534-40.

17. Valentonyte R, Hampe J, Huse K, Rosenstiel P, Albrecht M, Stenzel A, et al. Sarcoidosis is associated with a truncating splice site mutation in BTNL2. Nat Genet. 2005; 37(4): 357-364.
18. Schutt AC, Bullington WM, Judson MA. Pharmacotherapy for pulmonary sarcoidosis: a Delphi consensus study. Respir Med. 2010; 104(5): 717-723.

19. Chan ES, Cronstein BN. Molecular action of methotrexate in inflammatory diseases. Arthritis Res. 2002; 4(4): 266-273.

20. Marques LJ, Zheng L, Poulakis N, Guzman J, Costabel U. Pentoxifylline inhibits TNF-alpha production from human alveolar macrophages. Am J Respir Crit Care Med. 1999; 159(2): 508511

21. Kwak B, Mulhaupt F, Myit S, Mach F. Statins as a newly recognized type of immunomodulator. Nat Med. 2000; 6(12): 1399-1402.

22. Baughman RP, Winget DB, Lower EE. Methotrexate is steroid sparing in acute sarcoidosis: results of a double blind, randomized trial. Sarcoidosis Vasc Diffuse Lung Dis. 2000; 17(1): 60-66.

23. Baughman RP, Lower EE. Newer therapies for cutaneous sarcoidosis: the role of thalidomide and other agents. Am J Clin Dermatol. 2004; 5(6): 385-94. 